\section{Recurrent Subdural Hematomas in Benign Macrocrania of Infancy}

\section{Ademar Lucas Junior \\ São Camilo Diagnostic Medicine, Maringá, Brazil}

Corresponding author: Ademar Lucas Junior

São Camilo Diagnostic Medicine, Maringá, Paraná, Brazil.

\section{झ ademarlucasjr@gmail.com}

Tel: 5511992933038

Citation: Junior AL. Recurrent Subdural Hematomas in Benign Macrocrania of Infancy. Med Clin Rev. 2016, 2:3.

\section{Introduction}

Bening Macrocrania of Infancy (BMI) is a self-limited clinical entity [1-6] that affects approximately $5 \%$ of the pediatric population $[3,6]$. This condition has been known for a relatively short period of time, but it has a well-recognized clinical-radiologic correlation. $M R I$ is the imaging method of choice for its diagnosis $[2,6,7]$.

Its main characteristic is increased head circumference with preserved neuropsychomotor development. Radiologically, it is characterized by enlarged CSF spaces, notedly in frontotemporal regions, with no compression of the brain surface. The disproportion between cranial and brain volumes results in greater intracranial brain mobility, similar to what can be seen in elderly patients with cerebral atrophy. Both conditions lead to a greater likelihood of vascular rupture in subdural spaces and a consequent increased incidence of small subdural hematomas.
In children who are not affected by cerebral atrophy, this can be caused by minor head traumas. It is, therefore, imperative to identify the relation between the findings in order to prevent misdiagnosis.

\section{Case Report}

An 18-month-old boy, born via cesarean section at 31 weeks from a twin gestation, stayed in the PICU for 55 days due to pulmonary immaturity.

In his first year of life, he was submitted to bowel surgeries (appendectomy with ileostomy, and intestinal reconstruction in a two-stage procedure), and achieved full functional recovery.

He evolved with normal neuropsychomotor development and, at the end of his first year of life; an increased head circumference was identified. 
An MRI (Essenza, Siemens 1, 5T, Germany) of the skull was conducted when the patient was 13 months old and showed an enlarged subarachnoid space in the fronto-temporal regions, and no brain compression. Imaging findings characterized a right parafalcine parietal subdural hematoma adjacent to the superior sagittal sinus, without significant compression of the brain parenchyma (Figure 1). The parents denied any history of significant head trauma.

Five months later, a new MRI was conducted, demonstrating complete and spontaneous resolution of the right parietal subdural hematoma, and the appearance of a new left parietal hematoma, also adjacent to the superior sagittal sinus (Figure 2). Once again, there was no history of head trauma.

Funduscopic examination was normal. Lab tests, including coagulation tests, did not show any significant deviations either.

\section{Discussion}

$\mathrm{BMI}$ is a clinical entity defined as a quick and abnormal growth of the head circumference, usually above the $95^{\text {th }}$ percentile, in infants from 2 to 4 months of age [8].
Their cranial growth rate usually stabilizes at around 10-18 months of age [8] and recedes after the child completes 2 years of age [6], with only a minority of patients keeping an increased circumference after that.

The condition has been referred to by different names throughout the years, such as benign external hydrocephalus, idiopathic external hydrocephalus, and benign extra-axial collections of infancy $[1-5,8]$.

Its pathophysiology is still unknown [1] but the most widely accepted theory is that the condition is caused by immaturity of the arachnoid granulations $[1,2,4,5,7]$ resulting in impaired CSF reabsorption and a consequent fluid accumulation in extraaxial spaces and ventricles [2]. Some authors also associate this impaired reabsorption with an increased CSF production by the ventricles. These patients usually stabilize and even present a spontaneous macrocrania regression that coincides with the maturation of the arachnoid granulations, at around 2 years of age [2].

Some studies report a genetic predisposition for this condition, stating that people with family members who have an increased head circumference also have a greater likelihood of presenting

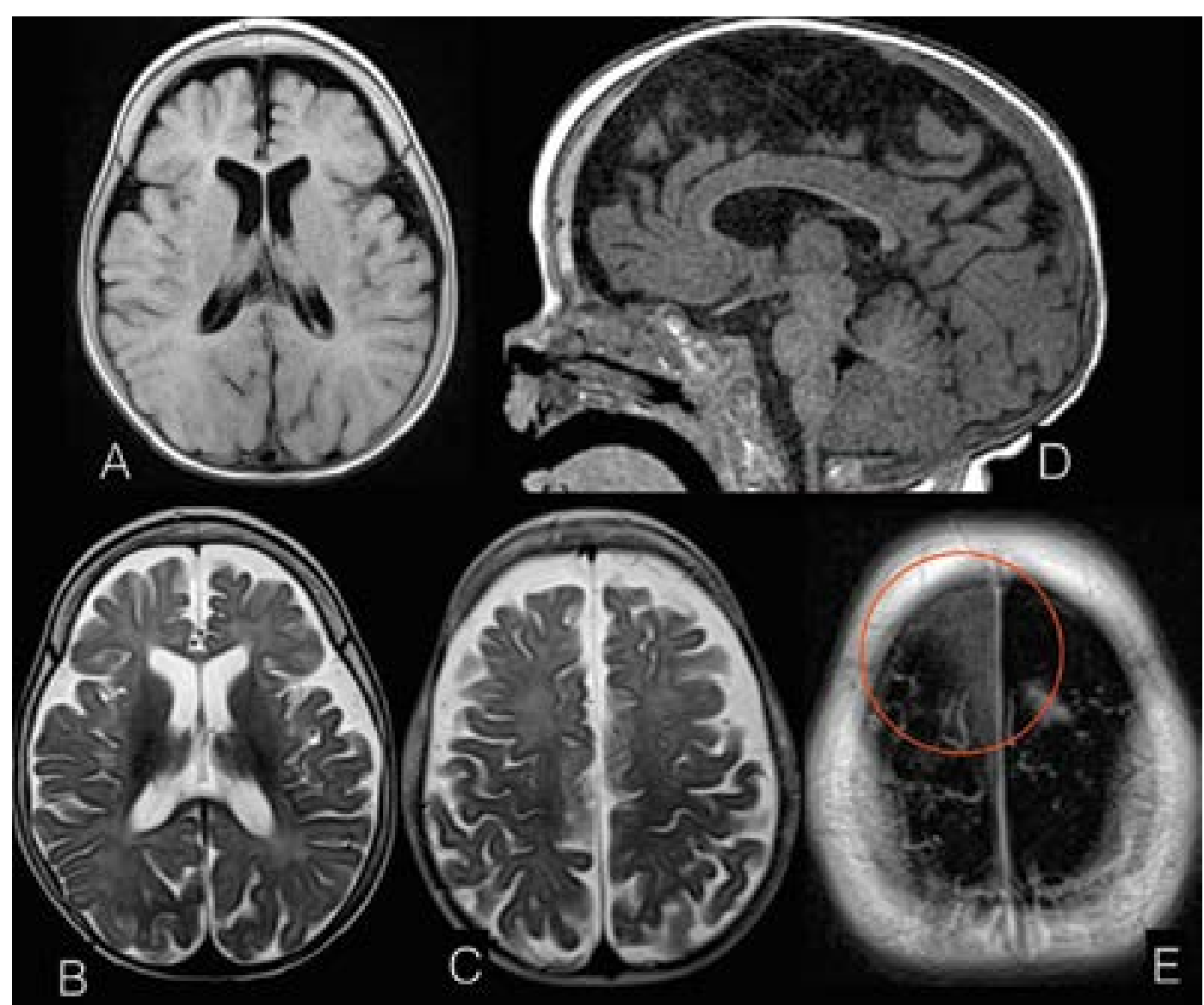

Figure 1 Axial T1 FSE (A) and T2 FSE (B e C) sequences show a prominent subarachnoid space in the frontal and temporal regions, without significant compression of the brain parenchyma. Sagittal T1 FSE (D) sequence shows craniofacial disproportion, with dominance of the skull over the face, corroborating the clinical finding of an increased head circumference. Axial FLAIR (E) sequence demonstrates a fluid collection with intermediate signal intensity in the right parafalcine subdural region, without significant compression of the brain's right hemisphere. 


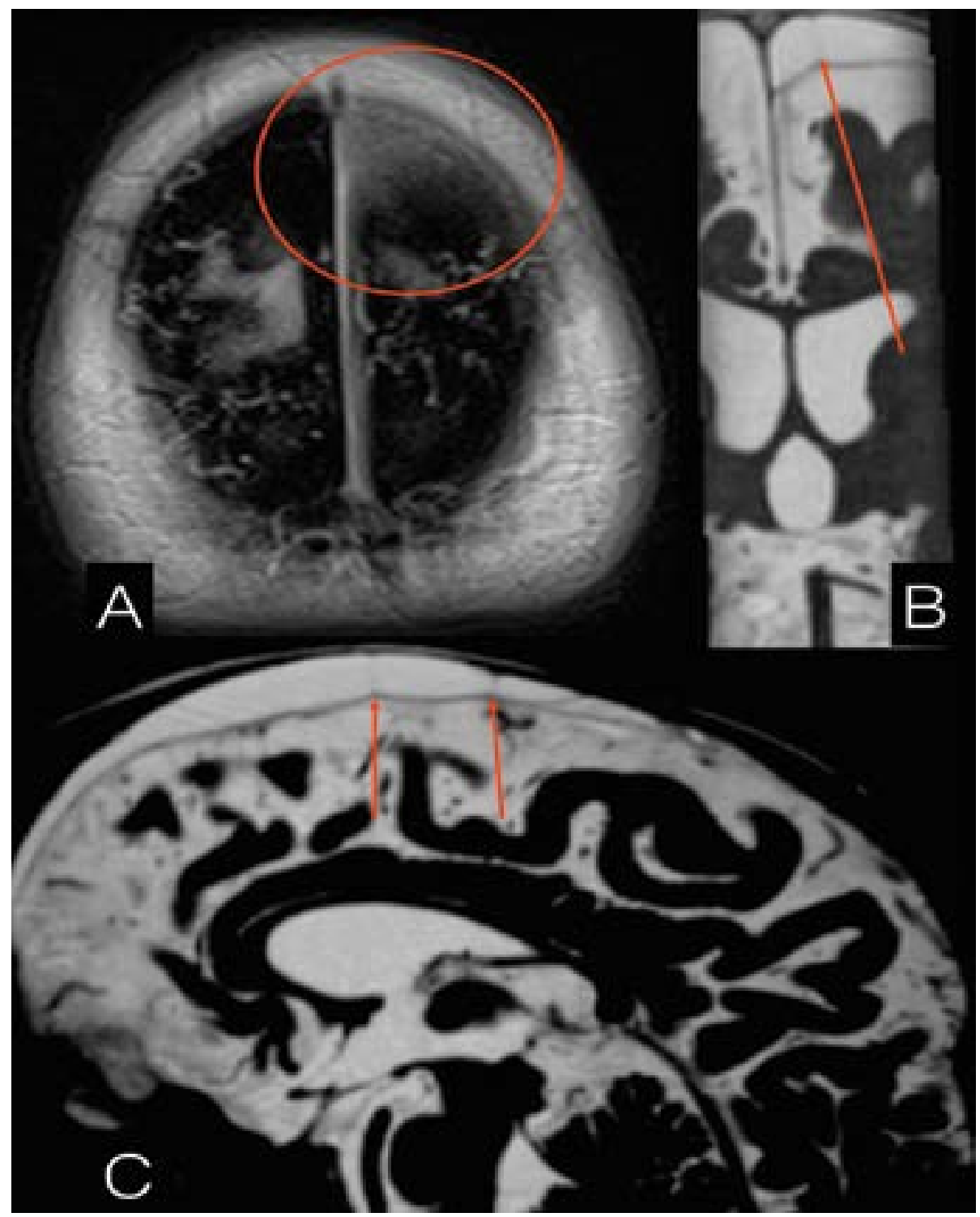

Figure 2 Axial FLAIR (A) sequence demonstrates complete and spontaneous resolution of the right parietal subdural hematoma and the appearance of a left fronto-parietal hematoma (circle). 3D CISS sequence with multiplanar reconstruction (coronal-B and sagittal-C) shows the described fluid collection, as well as stretching of the bridging veins draining into the superior sagittal sinus (arrows).

it $[1,2,4,6]$. There are also reports of a greater incidence of this condition in premature babies $[2,6]$ and in male patients $[1,8]$ accounting for up to two thirds of cases [2].

Most studies report assymptomatic patients with preserved neuropsychomotor development, hence its benign designation. However, there are studies that report a degree of neuropsychomotor development delay in some patients $[2,8]$ mainly affecting gross motor skills [1] speech development, and behavioral disorders [1]. According to some studies, however, these symptoms usually normalize by the second year of life [1] and according to others, by the forth year of life [2]. Reports of persistency of those delays are rare.

A few studies tried to compare the conservative therapy with surgical intervention and their possible repercussions on the patients' long-term neuropsychomotor development [2] but they included only a small sample of patients. Therefore, the description of a benign, self-limited entity still prevails in the literature, as well as the maintenance of the conservative therapy [2-4].

The diagnosis of the clinical manifestation is based on physical examination (head circumference and neuropsychomotor development of the child), and on imaging tests, especially MRI, as previously mentioned.

Imaging tests show CSF accumulation in extra-axial spaces, especially in the frontal regions of the skull (as compared to the posterior ones), sylvian fissure and anterior interhemispheric falx, associated with larger ventricles-though this is usually not very pronounced, and may range from mild to moderate [1-4,8].

A higher incidence of subdural hematomas may be spontaneous or may appear after minor head traumas [1,2,7-9] which cause the stretching of the bridging veins draining into the sagittal sinus, 
making them more susceptible to tearing and bleeding $[1,2,4,7]$ just like subdural hematomas in elderly patients with diffuse cerebral atrophy. Being aware of this possible complication is key, as subdural hematomas of infancy should raise suspicion of abuse child, especially when the child has hematomas in parafalcine regions and in different stages of evolution. In those cases, it is mandatory to investigate the presence of other characteristics of maltreatment victims, such as retinal petechial hemorrhage, encephalopathy and bone fractures in atypical locations [10]. A detailed medical history analysis is also critical [7].

Diagnosing subdural hemorrhage in children younger than 2 years of age is important as it may lead to harmful consequences such as seizures, focal neurologic deficits, developmental delay, and even death [1].

\section{Conclusion}

Benign Macrocrania of Infancy is a self-limited clinical entity that may lead to complications [1-5,8]. As such, it is extremely important that its association with subdural hematomas be acknowledged so that these children can be followed-up on and possible complications of this type of bleeding can be avoided [1]. An accurate diagnosis of this condition is crucial as its main differential diagnosis-non-accidental intracranial hemorrhage resulting from maltreatment-may have severe social, physical and mental health impacts on patients and families alike. 


\section{References}

1 McKeag H, Christian CW, Rubin D, Daymont C, Pollock AV, et al. (2013) Subdural hemorrhage in pediatric patients with enlargement of the subarachnoid spaces. J Neurosurg Pediatr 11: 438-444.

2 McNeely PD, Atkinson JD, Saigal G, O'Gorman AM, Farmer JP (2006) Subdural Hematomas in Infants with Benign Enlargement of the Subarachnoid Spaces Are Not Pathognomonic for Child Abuse. Am J Neuroradiol 27: 1725-1728.

3 Zahl SM, Egge A, Helseth E, Wester K (2011) Benign external hydrocephalus: a review, with emphasis on management. Neurosurg Rev 34: 417-432.

4 Medina LS, Frawley K, Zurakowski D, Buttros D, DeGrauw AJC, et al. (2001) Children with Macrocrania: Clinical and Imaging Predictors of Disorders Requiring Surgery. AJNR 22: 564-570.

5 Kuruvilla LC (2014) Benign enlargement of sub-arachnoid spaces in infancy. J Pediatr Neurosci 9: 129-131.
6 Suara RO, Trouth AJ, Collins M (2001) Benign Subarachnoid Space Enlargement of Infancy. J Natl Med Assoc 93: 70-73.

7 Wittschieber D, Karger B, Niederstadt T, Pfeiffer H, Hahnemann ML (2015) Subdural Hygromas in Abusive Head Trauma: Pathogenesis, Diagnosis, and Forensic Implications. Am J Neuroradiol 36: 432-439.

8 Marino MA, Morabito R, Vinci S, Germanò A, Briguglio $M$, et al. (2014) Benign External Hydrocephalus in Infants: A Single Centre Experience and Literature Review. Am J Neuroradiol 27: 245-250.

9 Feldman KW, Sugar NF, Browd SR (2015) Initial clinical presentation of children with acute and chronic versus acute subdural hemorrhage resulting from abusive head trauma. J Neurosurg Pediatr 16: 177185.

10 Miller D, Barnes P, Miller M (2015) The Significance of Macrocephaly or Enlarging Head Circumference in Infants With the Triad-Further Evidence of Mimics of Shaken Baby Syndrome. Am J Forensic Med Pathol 36: 111-120. 